\title{
Coping Strategies and Subjective Well-being: Context Matters
}

\author{
Rabea Fischer $^{1} \cdot$ Jakob Scheunemann $^{1}$ (D) . Steffen Moritz ${ }^{1}$
}

Accepted: 8 February 2021 / Published online: 6 March 2021

(C) The Author(s) 2021

\begin{abstract}
A growing body of research suggests that the functionality of coping strategies may in part depend on the context in which they are executed. Thus far, functionality has mostly been defined through the associations of coping strategies with psychopathology, particularly depression. Whether associations of coping strategies with proxies for happiness such as subjective well-being (SWB) are simply inverse remains to be shown. A total of $n=836$ individuals from the U.S. general population participated in an online survey that included a revised version of the Maladaptive and Adaptive Coping Styles Questionnaire (MAXR) that incorporates context-specific items, the Scale of Positive and Negative Affect (SPANE), the Temporal Satisfaction with Life Scale (TSWLS), the Patient Health Questionnaire (PHQ-9), and the Web Screening Questionnaire (WSQ). The MAX-R was submitted to an exploratory factor analysis. The factor analysis of the MAX-R yielded four subscales: adaptive, maladaptive, avoidance, and expressive suppression coping. Similar strategies in different contexts at times loaded on the same (e.g., maladaptive) or different (e.g., adaptive and avoidance) dimensions. Hierarchical multiple linear regression revealed significant associations of adaptive coping with SPANE $(\beta=0.21)$, TSWLS $(\beta=0.03)$, and PHQ-9 $(\beta=0.07)$, all $p s<.001$, of maladaptive coping with SPANE $(\beta=-0.19)$, TSWLS $(\beta=-0.10)$, and PHQ-9 $(\beta=0.02)$, all $p s<.01$, of avoidance with PHQ-9 ( $(=$ $0.01, p<.001)$, and of expressive suppression with SPANE $(\beta=-0.06)$ and TSWLS $(\beta=-0.16)$, $p s<.005$. Final models explained $64.6 \%$ of variance in SPANE, $41.8 \%$ of variance in TSWLS, and 55\% of variance in PHQ-9 score. In some instances, the functionality of coping strategies appears to be impacted by contextual factors. When investigating the overall benefit of use versus nonuse of coping strategies, their association with psychopathology measures and with subjective well-being should both be considered.
\end{abstract}

Keywords Subjective well-being · Happiness · Coping strategies · Mental health · Depression

Rabea Fischer

r.fischer@uke.de

1 Department of Psychiatry and Psychotherapy, University Medical Center Hamburg-Eppendorf, Martinistr. 52, 20246 Hamburg, Germany 


\section{Introduction}

For decades, psychological disorders were viewed as the result of an interplay between vulnerability and stress, thus assigning the individual a rather passive role. In the 1980s, Lazarus and Folkman (1984) highlighted the role of coping, a term which in recent times has been used interchangeably with emotion regulation. A recent model (Gross 2015) posits that coping predominantly pertains to the alleviation of stress responses, while emotion regulation is geared more closely toward influencing the emotion trajectory. A plethora of research has demonstrated that individuals with and without psychological disorders respond to stress differently on various levels, including psychophysiology (e.g., Alvares et al. 2016; Schiweck et al. 2019).

Research distinguishes between adaptive and maladaptive coping with stress. Mindfulness and cognitive reappraisal are commonly categorized as the former, while rumination, thought suppression, and catastrophizing are customarily categorized as the latter. There is now ample evidence suggesting that maladaptive coping is especially toxic (e.g., Joormann and Stanton 2016; Liu and Thompson, 2017; Moritz et al. 2016, 2018; Westphal et al. 2017). In contrast, the lack of adaptive coping seems less relevant in the pathogenesis of psychological disorders (Aldao and Nolen-Hoeksema 2012; Moritz et al. 2016). However, this simple dichotomy has been challenged by studies showing that the context in which coping occurs matters (Aldao 2013; Aldao and Tull 2015; Moritz et al. 2016). For instance, Rottweiler et al. (2018) have shown that suppression has different effects on anxiety depending on context; suppressing exam-related anxiety was associated with improved mood, whereas suppressing non-exam-related anxiety was not. Whether other strategies generally conceptualized as maladaptive show varying context-dependent associations with psychopathology needs to be explored further.

One instrument devised specifically for the investigation of the adaptiveness versus maladaptiveness of coping strategies is the Maladaptive and Adaptive Coping Styles Questionnaire (MAX; Moritz et al. 2016). It is comprised of pairs of items, where each item is phrased to reflect a supposedly adaptive or maladaptive behavior. It has been used to analyze coping patterns in, for example, depression and obsessive-compulsive disorder (Moritz et al. 2016, 2018). Previous results suggest that maladaptive coping and adaptive coping are not the exact inverse of each other and that associations among the subscales differ between clinical and nonclinical samples (Moritz et al. 2018).

\subsection{Subjective Well-Being}

Poor subjective well-being (SWB)—-defined as "the extent to which a person believes or feels his or her life is going well” (Diener et al. 2018, p. 1) - has been observed in various psychiatric disorders, such as depression (Baselmans et al. 2018), psychosis (Broyd et al. 2016; van Dijk et al. 2019), and gambling disorder (Farrell 2018). Seligman (2018), the founder of positive psychology, has emphasized that reducing or eliminating negative impacts of psychopathology, for example, does not inevitably result in elevated well-being. Similarly, Keyes (2005) has proposed a complete state model of health. According to this model, health and illness are not extreme poles on one bipolar dimension but rather two unipolar dimensions that correlate. Thus, individuals can be classified as completely mentally ill (presence of mental illness and absence of mental health), languishing (absence of mental health), flourishing (presence of mental health), or completely mentally healthy 
(absence of mental illness and presence of mental health). Complete mental health is associated with a variety of favorable outcomes such as low helplessness and high goals, resilience, and intimacy. However, it is also rare and has been found in only $17 \%$ of a representative U.S. population sample (Keyes 2005). Consequently, the absence of (dysfunctional) coping strategies that coincide with psychopathology does not automatically predict increased SWB. We also sought to explore whether a high use of presumably adaptive strategies directly translates into high SWB.

Thus far, few studies have assessed the relationship between coping strategies and SWB in the general population. One recent study investigated the correlations of SWB with the frequency and perceived efficacy of coping strategies for general affect regulation and for sadness regulation specifically (Korpela et al. 2018). The authors found varying but generally positive associations with SWB for positive activities, positive thinking, problemdirected action, and cognitive reappraisal. Similar associations were found in an Asian population in which positive reinterpretation, active coping, and planning were among the coping strategies positively correlated with life satisfaction and positive affect, whereas mental disengagement, denial, and behavioral disengagement were negatively associated with SWB measures (Chang et al. 2020). Nonetheless, many other strategies' associations with SWB remain unexplored.

\subsection{Aims}

The present study pursued two goals. First, we aimed to examine a revised version of the Maladaptive and Adaptive Coping Scale (MAX), which we extended to incorporate contextual factors. This allowed us to test whether, for example, expressive suppression, a strategy considered to be maladaptive, may under some circumstances manifest as adaptive (e.g., hiding negative emotions in front of strangers). Second, we examined which coping styles contribute to SWB. As mentioned, research has established that certain maladaptive styles contribute to the formation and maintenance of psychological disorders. What remains to be investigated is whether high levels of SWB and high levels of depressiveness are associated with opposite engagement patterns of presumably adaptive and presumably maladaptive coping.

\section{Methods}

The study was approved by the University Medical Center Hamburg-Eppendorf's ethics committee for psychological studies (LPEK-0026).

\subsection{Participants}

Participants were recruited using Amazon's Mechanical Turk (MTurk) in February and March 2019. Inclusion criteria were age 18 years or older and an IP address from the United States. A total of 1000 participants started the survey; 986 completed it. To address concerns of inattention, participants were required to have approval rates on previous studies of 95 percent or higher (Buhrmester et al. 2018). Poor results on an explicit attention check item ("I was focused while taking part in this study," answered on a 7-point scale: "strongly disagree" through "strongly agree"; a score of $<5$ led to exclusion) or on an implicit attention check item adopted from Oppenheimer et al. (2009) led to the exclusion 
of presumably inattentive participants. Finally, participants who sped through the survey (taking less than $60 \%$ of the estimated completion time) were also excluded (Arditte et al. 2016). All exclusions were made blind to results. After removal of inattentive subjects, $n=$ 836 participants were eligible for the final analyses.

\subsection{Procedure}

Data collection took place within a larger study that included several other questionnaires and experimental paradigms unrelated to the present study. The estimated time to complete the entire study was $30 \mathrm{~min}$; participants received $\$ 2.50$ upon completion. All questionnaires were implemented using the online survey tool Qualtrics.

\subsection{Psychometric Measures}

Current Mood Check. A single item ("How do you feel right now?") was used to assess current mood. Participants answered by moving a slider below a smiley face from a neutral position up to two units either to the left or to the right to indicate negative or positive mood, respectively.

\subsubsection{Subjective Well-Being}

Scale of Positive and Negative Experience. To assess affect balance, we employed the Scale of Positive and Negative Experience (SPANE; Diener et al. 2010). The scale consists of 12 emotion adjective items, with six items assessing positive and six assessing negative affective experience over the previous four weeks. Items are endorsed on a 5-point frequency response scale ranging from $1=$ "very rarely or never" to $5=$ "very often or always." Subtracting the total negative score from the total positive score yields an affect balance measure, with high scores reflecting high SWB. The scale showed acceptable test-retest reliability ( $r=0.71)$ and good internal consistency (Chronbach's $\alpha=0.87$; Diener et al. 2010).

Temporal Satisfaction with Life Scale. The 15-item Temporal Satisfaction with Life Scale (TSWLS; Pavot et al. 1998) assesses life satisfaction in the past, present, and expected future. Items are endorsed on a 7-point Likert-scale ranging from $1=$ "strongly disagree" through $7=$ "strongly agree," where high total scores indicate high life satisfaction. Test-retest reliability was good $(r=0.82)$ and internal consistency excellent (Chronbach's $\alpha=0.91$ ) in a community sample (Pavot et al. 1998).

\subsubsection{Psychopathology}

Web Screening Questionnaire. To screen for a variety of psychiatric disorders, we used the Web Screening Questionnaire (WSQ; Donker et al. 2009). It comprises 21 self-rated items selected from reliable and valid instruments that assess common mental disorders. Although the WSQ has a rather high false positive rate (specificity from 0.63 to 0.80 ), it also shows high sensitivity $(0.72-1.00)$; criterion validity has been demonstrated in the general population (AUC range $=0.65-0.81$; van Ballegooijen et al. 2016).

Patient Health Questionnaire. The Patient Health Questionnaire (PHQ-9; Kroenke et al. 2001) was used to screen for depressive symptoms. This instrument consists of nine items inquiring about the frequency of depressive symptoms over the last two weeks plus one 
additional item assessing impairment due to depressive symptoms. It is a reliable and valid measure of depression in the general population (Kocalevent et al. 2013).

Previous Psychiatric Diagnoses. In the sociodemographic questionnaire, participants also disclosed whether they had previously received a psychiatric diagnosis and, if yes, which diagnosis.

\subsubsection{Coping Strategies}

Maladaptive and Adaptive Coping Styles Questionnaire Revised. For the present study, we supplemented the established 21-item Maladaptive and Adaptive Coping Styles Questionnaire (MAX; Moritz et al. 2016) with 20 additional items (MAX-R). The original MAX measures coping styles along three subscales: maladaptive, adaptive, and avoidance. It is comprised of one individual and 10 pairs of opposite items, one representing the adaptive and one the maladaptive behavior (e.g., "I try to quickly stop fruitless rumination" vs. "I am prone to rumination"). Items are endorsed on a 4-point Likert scale from $1=$ "not true" to $4=$ "true." Its internal consistency is good for the adaptive and maladaptive subscales (Chronbach's $\alpha=0.87$ resp. Chronbach's $\alpha=0.85$ ) but is lower for the avoidance subscale (Chronbach's $\alpha=0.65$; Moritz et al. 2016).

We followed the notion of Aldao (2013) that the adaptiveness of an emotional response needs to be judged within the context that elicits it and that adaptiveness may reflect a rather dynamic use of various strategies to achieve optimal levels of emotions in a given context. Several of the newly devised items were designed to reflect behavior in specific contexts and situations (e.g., "I hide my emotions when it is appropriate (e.g., when getting negative feedback from my boss)"; "I try to suppress negative thoughts in situations in which having such thoughts is not useful (such as when giving a speech)").

\subsection{Statistical Analysis}

All analyses were performed in SPSS (IBM Corp., Version 25). First, we submitted the MAX to an exploratory factor analysis with orthogonal (varimax) rotation. Second, Pearson correlation coefficients were calculated for study variables. Finally, we performed hierarchical multiple linear regression using forced entry to predict (a) affect balance, (b) life satisfaction, and (c) depressiveness (as measured by the PHQ-9). In the first step of the hierarchical model, we entered sociodemographic data (age, gender, education, ethnicity), psychiatric diagnoses, and current mood. When predicting (a) affect balance and (b) life satisfaction, we also entered depressiveness as a predictor in the first step of the model; when predicting (c) depressiveness, we also included affect balance and life satisfaction as predictors in the first step of the model. In the second step (final model) we added the four coping subscales as well as depressiveness for the outcomes (a) affect balance and (b) life satisfaction, and SWB measures for (c) depressiveness.

As previous research has focused on the genders of male and female, we only investigated gender differences between males and females ("binary gender") and excluded four participants from the analysis who did not identify as either male or female. Education was operationalized as three dummy variables comparing (1) started but incomplete college degree, (2) completed college degree, and (3) professional degree to high school or less. As the present sample consisted mostly of individuals identifying as White/European American, we entered ethnicity as a binary variable (White vs. non-White). Psychiatric diagnoses were entered as two dichotomous variables. The first variable reflected whether 
a participant had screened positive for at least one psychiatric diagnosis on the WSQ; the second indicated whether a participant had previously been diagnosed with a psychiatric illness, according to self-report.

\section{Results}

\subsection{Demographic Data}

Mean age of participants was $40.3(S D=12.6)$ years with a range of 18 to 75 years. Almost all participants were native English speakers (96.9\%) and U.S. citizens (98.2\%) according to self-report. Slightly more than half of the sample (56.2\%) identified as female; $0.4 \%$ identified as neither male nor female. Overall, $0.6 \%$ of participants had completed less than a high school degree, $11.1 \%$ had graduated high school, $24.4 \%$ had started college, $48.6 \%$ had a college degree, and $15.3 \%$ had a professional degree or doctorate. More than threequarters of the participants (78.0\%) identified as White/European American (Black/African American: 11\%, Latino/Hispanic: 6.3\%, Asian/Asian American: 5.7\%, Native American/ Hawaiian: $1.9 \%$, other: $0.6 \%$; multiple answers possible). In the sociodemographic questionnaire, $54.8 \%$ of all participants stated that they had not previously received a psychiatric diagnosis. The numbers of participants who had received psychiatric diagnoses is displayed in Table 4 in the Appendix. Table 4 also shows the numbers of participants who screened positive for psychiatric disorders using the WSQ. According to the PHQ-9, 24.2\% of participants were classified as currently depressed when using a cut-off value of 10 as recommended by Manea et al. (2012).

On average, participants displayed more positive than negative affect (mean affect balance score $=7.63, S D=9.23$; one-sample $t$-test against midpoint: $t(834)=23.874, p<.001$ ) and moderate life satisfaction (mean score $=62.83, S D=20.46$ ). Mean depressiveness (PHQ-9) score was $6.17(S D=6.21)$, reflecting mild (subclinical) depressive symptoms (Kroenke and Spitzer 2002).

\subsection{Exploratory Factor Analysis}

The factor analysis of the MAX-R resulted in seven factors with an eigenvalue greater than 1. Based on the scree plot inflections, four factors were retained, explaining $43.9 \%$ of total variance. The first (14.85\% variance explained), second (12.58\%), and third (8.62\%) factors resembled the adaptive, maladaptive, and avoidance scales reported by Moritz et al. (2016) and were named accordingly. The fourth factor (7.85\%) was labeled "expressive suppression" as it was comprised only of variants of suppressing outward emotional expression, which is referred to as expressive suppression (Dryman and Heimberg 2018). Each of the four factors was used as a subscale in the final questionnaire. All items with loadings of 0.5 or higher were included in the respective subscale. The full pattern matrix is displayed in Table 1. Context-related allocations are described in Sect. 3.3.

Two previously (according to Moritz et al. 2016) adaptive and one previously maladaptive item showed factor loadings below 0.5 and were subsequently not allocated to a subscale. None of the three items allocated to the avoidance subscale by Moritz et al. (2016) remained part of it; two of the items were then categorized as expressive suppression while one item's loading did not exceed 0.5 for avoidance. Several of the newly included items did not exceed loadings of 0.5 for any factor. 


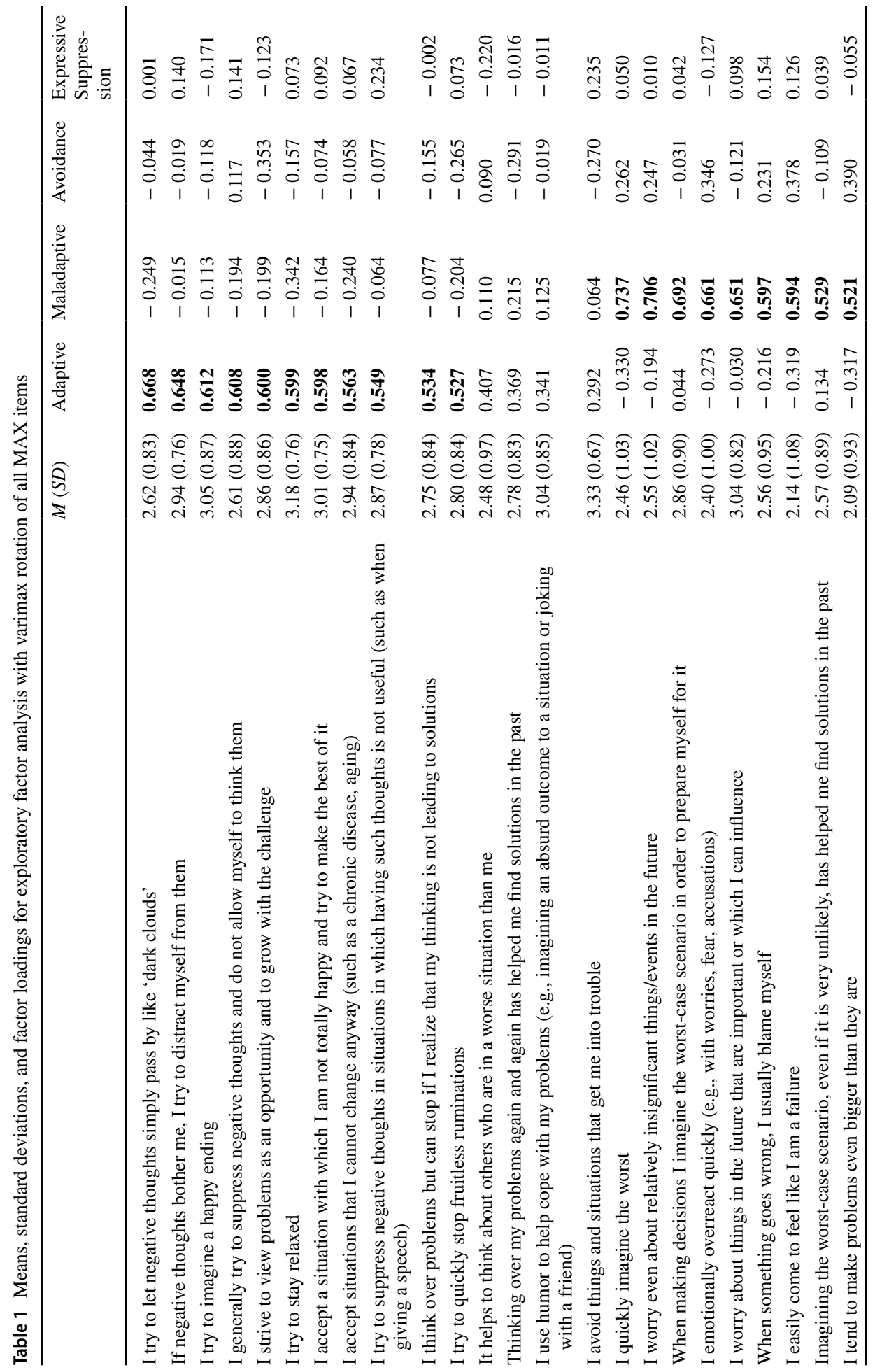




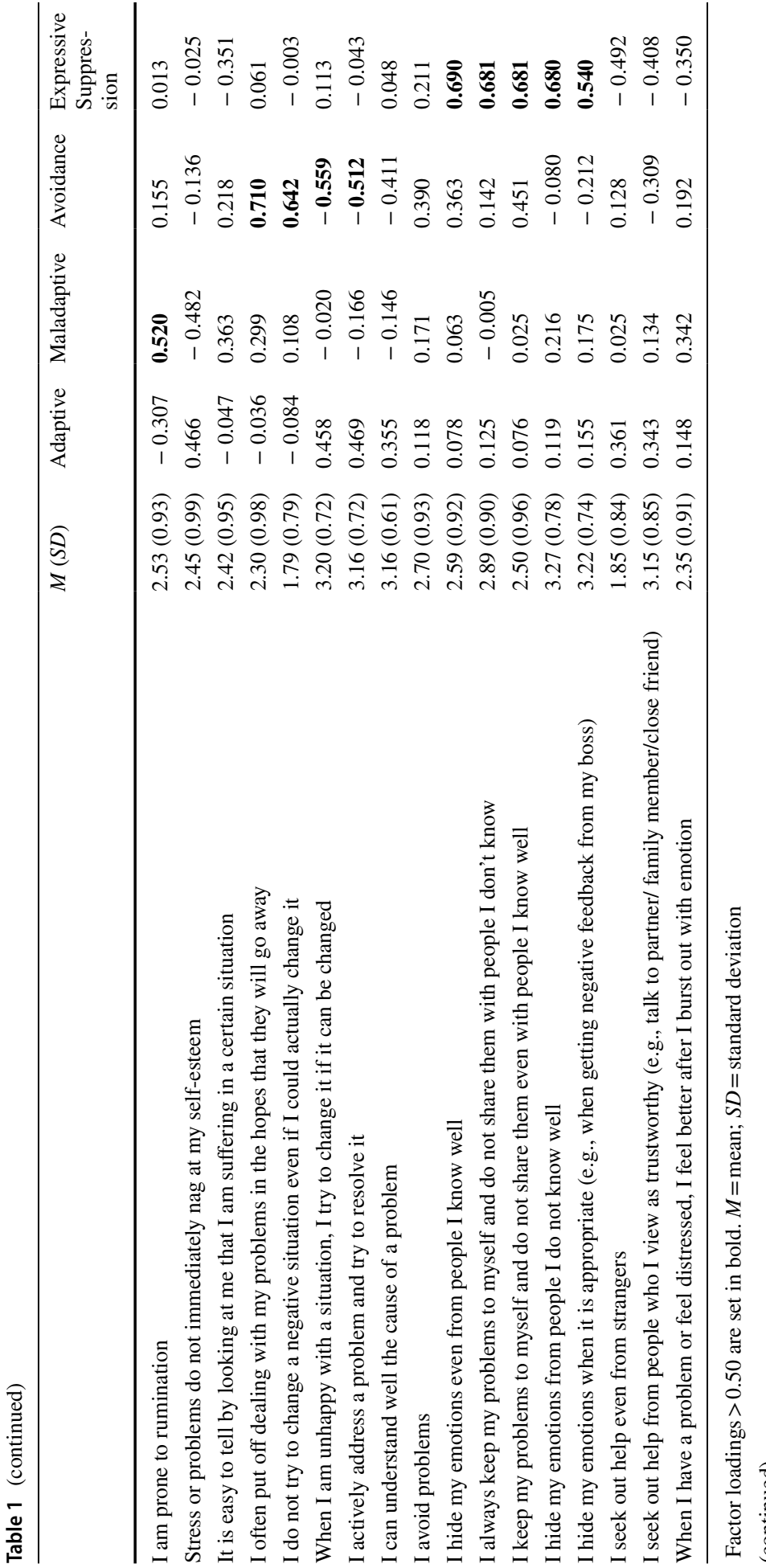


Table 2 Bivariate correlations of coping subscales with each other, SWB measures, and depressiveness

\begin{tabular}{|c|c|c|c|c|c|c|}
\hline & Maladaptive & Avoidance & $\begin{array}{l}\text { Expressive } \\
\text { suppression }\end{array}$ & Affect balance & $\begin{array}{l}\text { Life satisfac- } \\
\text { tion }\end{array}$ & Depressiveness \\
\hline Adaptive & $-0.511 * * *$ & $-0.485 * * *$ & $0.106^{* *}$ & $0.535 * * *$ & $0.456 * * *$ & $-0.372 * * *$ \\
\hline Maladaptive & & $0.496 * * *$ & $0.156^{* * * *}$ & $-0.599 * * *$ & $-0.409^{* * *}$ & $0.594 * * *$ \\
\hline Avoidance & & & $0.141 * * *$ & $-0.419 * * *$ & $-0.280 * * *$ & $0.416^{* * * *}$ \\
\hline $\begin{array}{l}\text { Expressive } \\
\text { suppression }\end{array}$ & & & & $-0.148 * * *$ & $-0.174 * * *$ & $0.156^{* * * *}$ \\
\hline Affect balance & & & & & $0.654 * * *$ & $-0.649 * * *$ \\
\hline $\begin{array}{l}\text { Life satisfac- } \\
\text { tion }\end{array}$ & & & & & & $-0.411 * * *$ \\
\hline
\end{tabular}

$* * * p<.001, * * p<.01$

Internal consistency of the newly formed subscales was good for adaptive $(\alpha=0.86)$ and maladaptive $(\alpha=0.88)$ coping and acceptable for avoidance coping $(\alpha=0.73)$ and expressive suppression $(\alpha=0.78)$. The latter two subscales show improved reliability compared to the original MAX's avoidance subscale $(\alpha=0.65$; Moritz et al. 2016). Inter-item correlations of subscales ranged from $r=0.512$ for adaptive, from $r=0.120$ to 0.667 for maladaptive, from $r=0.280$ to 0.487 for avoidance, and from $r=0.338$ to 0.751 for expressive suppression. Six items had commonalities lower than 0.4 and were therefore not included in any subscale: "Thinking over my problems again and again has helped me find solutions in the past," "I use humor to help cope with my problems (e.g., imagining an absurd outcome to a situation or joking with a friend)," "I avoid things and situations that get me into trouble," "It is easy to tell by looking at me that I am suffering in a certain situation," "I avoid problems," and "When I have a problem or feel distressed, I feel better after I burst out with emotion."

Mean item endorsement scores were highest for expressive suppression (2.89, $S D=0.62)$, followed by adaptive $(2.88, S D=0.53)$, maladaptive $(2.52, S D=0.66)$, and avoidance $(1.93, S D=0.60)$ coping. Bivariate correlations of coping subscales, SWB measures, and depressiveness can be found in Table 2 .

\subsection{Situational Dependence of Coping Strategies' Functionality}

Some items in the MAX-R were intended to capture the same strategy but represent different contexts. Yet, the factor loadings of these items often did not differ. For example, all items on catastrophizing loaded on the factor maladaptive, notwithstanding different contexts ("When making decisions I imagine the worst-case scenario in order to prepare myself for it"; "Imagining the worst-case scenario, even if it is very unlikely, has helped me find solutions in the past"). Items of the subscale "expressive suppression" all showed medium to high intercorrelations $(r=0.751)$, and therefore whether someone hides emotions or problems from individuals they know well versus those they do not know well did not impact the categorization based on factor loadings in this sample. Accordingly, in such cases items were pooled as one subscale. However, some strategies' categorization depended on wording: "I accept a situation with which I am not totally happy and try to make the best of it" was categorized as adaptive, while "When I am unhappy with a situation, I try to change it if it can be changed" was classified as avoidant (inverse). Based on 
the factor loadings of the exploratory factor analysis, thought suppression, which is usually classified as an avoidance strategy, was categorized as adaptive, represented by the two items "I generally try to suppress negative thoughts and do not allow myself to think them" and "I try to suppress negative thoughts in situations in which having such thoughts is not useful (such as when giving a speech)."

In addition, participants' current mood impacted associations between SWB, depressiveness, and adaptive, maladaptive, and avoidance coping. Accounting for current mood reduced the associations of the adaptive coping subscale with affect balance, satisfaction with life, and depressiveness by $0.125,0.125$, and 0.120 , respectively. Associations of maladaptive coping were reduced by $0.075,0.117$, and 0.067 , respectively, and those of avoidance coping by $0.083,0.105$, and 0.070 , respectively. Current mood did not impact the correlations of expressive suppression (respective reductions: 0.011, 0.006, 0.021). All correlations remained significant $(p<.001)$ after controlling for current mood.

\subsection{Associations between Coping, Subjective Well-Being, and Depressiveness}

\subsubsection{Affect Balance}

A hierarchical linear regression analysis was conducted to evaluate the prediction of affect balance based on depressiveness, binary gender, age, education, ethnicity, WSQ diagnosis, previous psychiatric diagnosis, current mood, and adaptive, maladaptive, avoidance, and expressive suppression coping. In step 1 of the analysis, the predictor variables depressiveness, gender, age, education, ethnicity, WSQ diagnosis, previously received psychiatric diagnosis, and current mood were added. The model was significant $(F(10,809)=107.61$, $\left.p<.001 ; R^{2}=0.571\right)$.

In step 2 of the analysis, the predictor variables adaptive, maladaptive, avoidance, and expressive suppression coping were added. The second model was also significant $\left(F(14,805)=105.12, p<.001 ; R^{2}=0.646\right)$ and improved the prediction by explaining an additional $7.6 \%$ of the variance in affect balance; this change in $R^{2}$ was significant $(F(4$, $805)=43.01, p<.001)$. In this second, final model, education, WSQ diagnosis, previous psychiatric diagnosis, and avoidance coping did not significantly predict affect balance; however, gender, age, ethnicity, current mood, depressiveness, adaptive coping, maladaptive coping, and expressive suppression coping did significantly predict values of affect balance. Women, younger individuals, people identifying as White, those who reported more positive current mood, those who reported less depressiveness, and those who engaged in more adaptive coping, less maladaptive and less expressive suppression coping showed higher levels of positive affect. Coefficients, confidence intervals, and $p$-values are displayed in Table 3.

\subsubsection{Satisfaction with Life}

A hierarchical linear regression analysis was conducted to evaluate the prediction of life satisfaction based on depressiveness, binary gender, age, education, ethnicity, WSQ diagnosis, previous psychiatric diagnosis, current mood, and adaptive, maladaptive, avoidance, and expressive suppression coping. In step 1 of the analysis, the predictor variables depressiveness, gender, age, education, ethnicity, WSQ diagnosis, previously received psychiatric diagnosis, and current mood were added. The model was significant $(F(10,809)=38.91$, $\left.p<.001 ; R^{2}=0.325\right)$. 


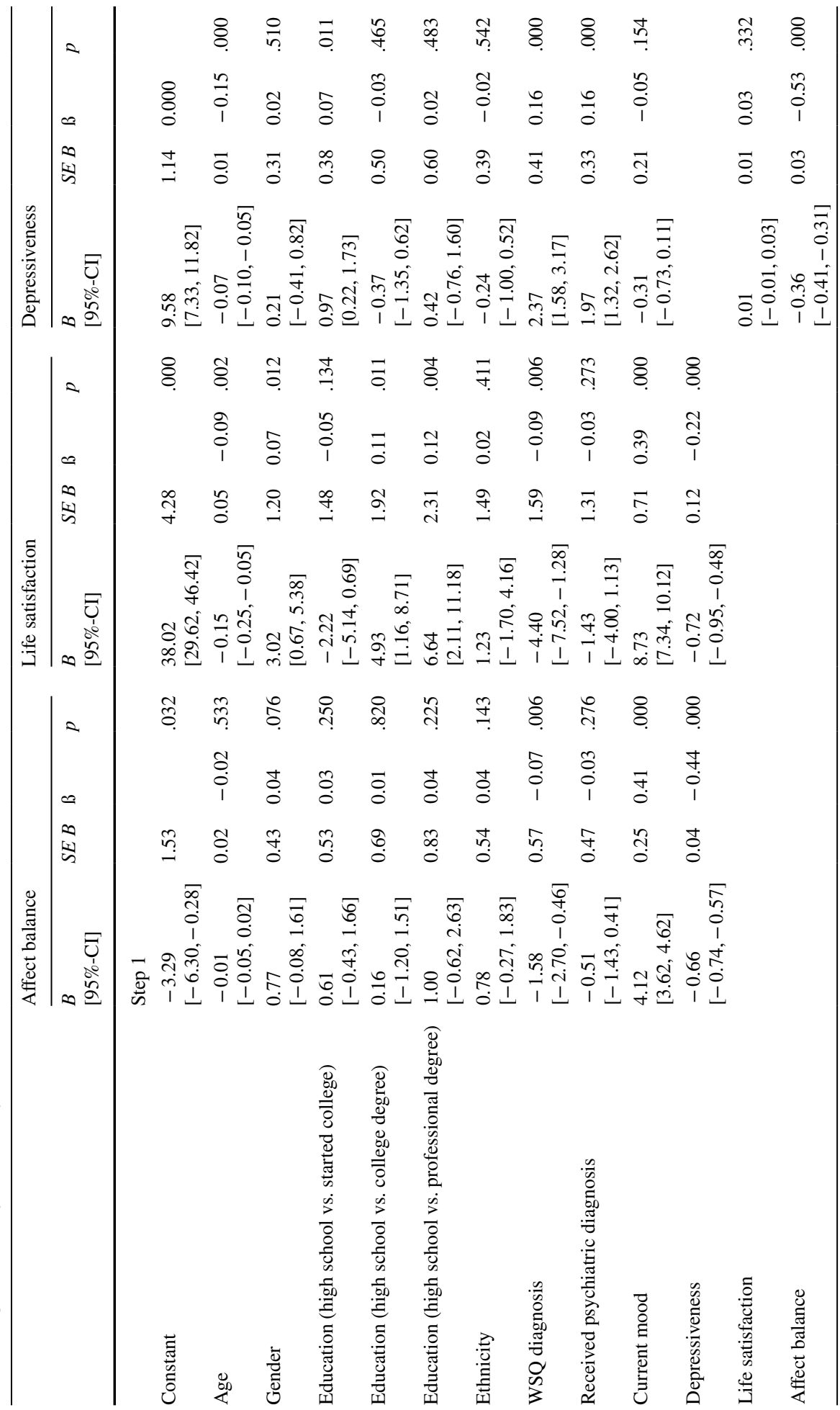




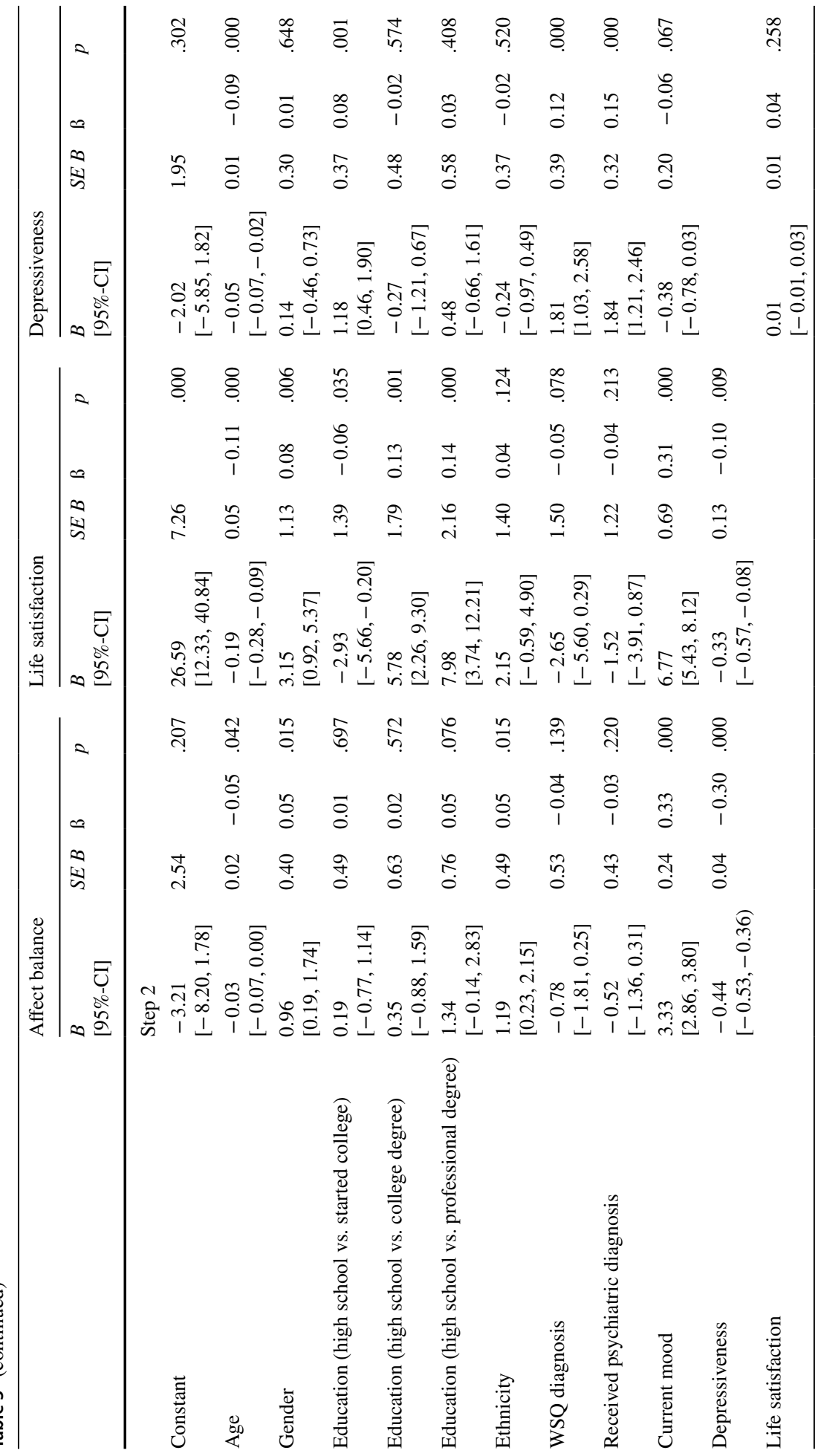




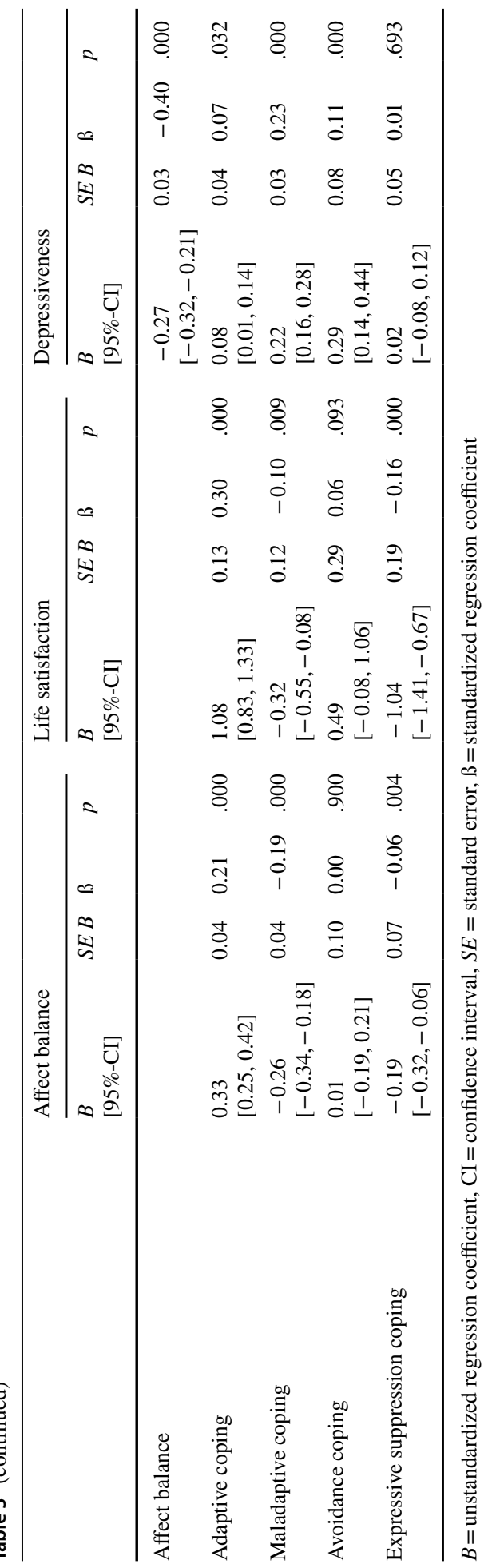


In step 2 of the analysis, the predictor variables adaptive, maladaptive, avoidance, and expressive suppression coping were added. The second model was also significant $(F(14$, $\left.805)=41.28, p<.001 ; R^{2}=0.418\right)$ and improved the prediction by explaining an additional $9.3 \%$ of the variance in life satisfaction; this change in $R^{2}$ was significant $(F(4$, $805)=32.20, p<.001)$. In this second, final model, ethnicity, WSQ diagnosis, previous psychiatric diagnosis, and avoidance coping did not significantly predict values of life satisfaction; however, gender, age, education, current mood, depressiveness, adaptive coping, maladaptive coping, and expressive suppression coping did significantly predict values of life satisfaction. Women, younger individuals, those with a college or professional degree compared to those with high school or less, those with high school or less compared to those who started but had not finished college, those who reported more positive current mood, those who reported less depressiveness, and those who engaged in more adaptive coping, less maladaptive and less expressive suppression coping showed higher levels of life satisfaction. Coefficients, confidence intervals, and $p$-values are displayed in Table 3.

\subsubsection{Depressiveness}

A hierarchical linear regression analysis was conducted to evaluate the prediction of depressiveness based on affect balance, life satisfaction, binary gender, age, education, ethnicity, WSQ diagnosis, previous psychiatric diagnosis, current mood, and adaptive, maladaptive, avoidance, and expressive suppression coping. In step 1 of the analysis, the predictor variables affect balance, life satisfaction, gender, age, education, ethnicity, WSQ diagnosis, previously received psychiatric diagnosis, and current mood were added. The model was significant $\left(F(11,808)=74.47, p<.001 ; R^{2}=0.503\right)$.

In step 2 of the analysis, the predictor variables adaptive, maladaptive, avoidance, and expressive suppression coping were added. The second model was also significant $(F(15$, $\left.804)=65.63, p<.001 ; R^{2}=0.550\right)$ and improved the prediction by explaining an additional $4.7 \%$ of the variance in depressiveness; this change in $R^{2}$ was significant $(F(4,804)=21.02$, $p<.001$ ). In this second, final model, gender, education (completed college or completed professional degree as compared to high school or less), ethnicity, current mood, expressive suppression coping, and life satisfaction did not significantly predict values of depressiveness; however, age, having started but not finished college as compared to having completed high school or less, WSQ diagnosis, previous psychiatric diagnosis, affect balance, adaptive coping, maladaptive coping, and avoidance coping did significantly predict values of depressiveness. Younger individuals, those who had started but not finished college, those who reported less positive affect, and those who engaged in more adaptive, more maladaptive and more avoidance coping showed higher levels of depressiveness. Coefficients, confidence intervals, and $p$-values are displayed in Table 3.

\section{Discussion}

The present study pursued two aims. First, using a newly developed instrument (the Revised Maladaptive and Adaptive Coping Styles Questionnaire, MAX-R), we explored whether context modulates the (dys)functionality of some coping strategies. Second, we investigated how coping strategies are associated with subjective well-being (SWB). 


\subsection{Summary of Findings}

The factor analysis of the MAX-R essentially replicated the previous structure yielding adaptive, maladaptive, and avoidance subscales (Moritz et al. 2016). The avoidance subscale then comprised new context-capturing items (e.g., "I do not try to change a negative situation even if I could actually change it"). We additionally identified a fourth subscale, which we labeled expressive suppression in accordance with the literature (e.g., Dryman and Heimberg 2018).

Bivariate correlations between coping subscales, SWB measures (affect balance and life satisfaction), and depressiveness emerged in the expected directions. Specifically, both affect balance and life satisfaction were significantly positively associated with adaptive coping and significantly negatively associated with all other coping subscales and depressiveness. Depressiveness was significantly negatively associated with adaptive coping and significantly positively associated with the other three coping subscales.

Multiple linear regression showed that-after the influence of sociodemographic variables, psychiatric diagnoses, current mood, and depressiveness was accounted for-adaptive, maladaptive, and expressive suppression coping, but not avoidance coping, contributed significantly to the prediction of affect balance and life satisfaction in the expected directions. After accounting for sociodemographic variables, psychiatric diagnoses, current mood, and SWB measures, adaptive, maladaptive, and avoidance coping, but not expressive suppression coping, significantly contributed to the prediction of depressiveness. All associations with depressiveness were in the expected direction with the exception of adaptive coping, which yielded a small positive contribution to depressiveness scores. In summary, differential patterns of small but significant contributions of coping to SWB and depressiveness emerged.

Of the covariates, gender significantly predicted SWB (but not depressiveness) in that women showed higher SWB scores than men. Younger age was associated with higher scores on all three outcomes. Higher education was associated with higher life satisfaction (but not affect balance). Individuals who had started but not finished college showed higher depressiveness than individuals who had completed high school or less; no differences were found for college or professional degree compared to high school degree. Participants who identified as White showed higher affect balance scores than participants who identified as non-White. Current mood predicted SWB in a positive direction but not depressiveness.

\subsection{Context-Dependent Functionality of Coping Strategies}

We added several items that put the coping strategies into different contexts to the MAX questionnaire to test whether some coping strategies are maladaptive in some contexts but adaptive in others. For some strategies, for example catastrophizing, all items loaded on the factor maladaptive regardless of context. Hence, catastrophizing appears to always be maladaptive. The former (MAX) and new (MAX-R) expressive suppression items (see Table 1) also did not present as differing in terms of adaptiveness. Instead of being part of the previously established adaptive versus maladaptive subscales, they now constitute a new subscale. This new subscale significantly contributed to the prediction of the two SWB measures (negative association) but not to the prediction of depressiveness.

Interestingly, acceptance appears to be less clear-cut in terms of functionality. We found that the original noncontextual acceptance item ("I accept a situation with which I am not 
totally happy and try to make the best of it") was replicated as adaptive. Yet, it appears that the question of whether one is actually able to influence the situation at hand plays a role in the functionality of the coping behavior. According to our data, accepting situations is most adaptive if the situation presumably cannot be changed. When it is clear that the individual does have a subjective influence over the unfavorable situation ("When I am unhappy with a situation, I try to change it if it can be changed"; "I do not try to change a negative situation even if I could actually change it"), then accepting the situation appears to represent an avoidance strategy and accordingly was not associated with SWB; instead, a weak association with depressiveness emerged. To conclude, our results essentially agree with previous work (e.g., Aldao 2013; English et al. 2017; Tang and Huang 2019) emphasizing the need to consider context factors when judging strategies' functionality. However, context seems to be of varying importance for different styles.

\subsection{Coping Strategies, Subjective Well-Being, and Depressiveness}

Overall, our findings speak to the hypothesis that well-being as an expression of mental health correlates with, but is not the polar opposite of, mental illness (Keyes 2005). We found differential associations of coping subscales with affect balance, life satisfaction, and depressiveness. Although maladaptive coping predicted SWB via negative associations $(B=-0.26, p<.001$ for affect balance; $B=-0.32, p=.009$ for life satisfaction) and depressiveness via a positive association $(B=0.22, p<.001)$ at similar magnitudes, the other coping subscales did not show inverse correlations with SWB and depressiveness as one would expect if SWB and depressiveness were at opposite ends of one bipolar construct.

We found that both adaptive and maladaptive strategy use predicts both SWB and depressiveness scores. However, avoidance coping was found to predict depressiveness but not SWB, while expressive suppression was found to predict SWB but not depressiveness. Numerically, adaptive coping has the highest explanatory value for life satisfaction, maladaptive coping has the highest explanatory value for depressiveness, avoidance coping has significant explanatory value only for depressiveness, and expressive suppression has significant explanatory value only for SWB (more so for life satisfaction than for affect balance).

Thus far, few studies have investigated the link between adaptive and maladaptive coping strategies and well-being. Our finding that adaptive strategies show a positive correlation and maladaptive strategies a negative correlation with aspects of SWB is in line with a previous study by Balzarotti et al. (2016). They found that positive reappraisal and catastrophizing in particular were strongly associated with SWB positively or negatively, respectively. Similar to the results of Moritz et al. (2016), we also found the positive correlations of maladaptive $(>0.5)$ and avoidance $(>0.4)$ coping to be numerically higher with depressiveness than the negative correlation of adaptive coping $(<-0.4)$. When accounting 
for sociodemographic factors, current mood, SWB measures, and the other coping subscales, the unique contribution of adaptive coping to depressiveness was a small positive contribution. The adaptive coping score is a sum score of endorsement for all items on this subscale. This result thus may reflect the previous finding that a higher total sum of the maladaptive and adaptive strategies used is associated with a higher depressiveness score (Moritz et al. 2016) and the conclusion that a larger repertoire of coping strategies does not directly translate to decreased depressiveness. A possible explanation is that people who use certain coping strategies ineffectively use a larger pool of strategies to compensate for the insufficient regulation; however this theory needs to be examined further. Overall, our findings concur with the suggestion that maladaptive and adaptive coping are not inverse and that addressing maladaptive coping seems more essential than enhancing adaptive coping for alleviating psychiatric symptoms (Moritz et al. 2016).

In summary, certain coping strategies, particularly cognitive reappraisal, seem to have the potential to contribute to SWB while others, particularly catastrophizing and expressive suppression, appear to undermine SWB. This appears to be an add-on effect to the aggravating or appeasing role coping strategies play in psychopathologies such as depression, which further supports the notion that well-being is more than the absence of psychopathology (Franken et al. 2018; Keyes 2007).

\subsection{Limitations}

We need to acknowledge several limitations. Due to the online design of our study, there are several aspects of the data-gathering process that we could not fully control, such as whether participants responded in a distraction-free environment. However, the online setting enabled us to recruit a large community sample and, as a precaution, we implemented several attention checks and additional measures to ensure good data quality (Buhrmester et al. 2018). In addition, we used self-report measures for the assessment of both coping strategies and SWB and our study design is cross-sectional, which does not allow for extended causal conclusions. Our results thus need to be corroborated by longitudinal designs, preferably using ecological momentary assessment. Analyzing our data, we further found that our sample was more highly educated (64\% college or professional degree) than the general population of the U.S. aged 18 and older, of whom about $43 \%$ held a post-high school degree in 2019 (U.S. Census Bureau 2020). As we accounted for education when conducting our analysis, we do not believe that this difference is a significant concern. However, future studies should differentiate the associations with coping and SWB across various levels of education. We also do not know for certain how relevant the context provided in the items was for the participants. Future studies should refine contexts and replicate findings, especially concerning items that were intended to distinguish between cognitive or emotional suppression with close social contacts versus strangers. Although the additional items were able to tease out only a few contextual differences, the revised version of the questionnaire in our opinion does provide additional value for future 
studies due to its improved internal consistency. Finally, in our investigation we aimed to differentiate between subjective well-being and depressiveness. We have to acknowledge that SWB most likely does not cover all aspects of mental health and that depressiveness, as measured by the PHQ-9, is only one facet of mental illness or psychopathology. However, as depressed mood occurs in various forms of mental illness, we believe that it is an appropriate measure for this exploratory study. Further studies comparing undiagnosed individuals to those who have verified diagnoses of mental disorders are needed to explore in more depth the differential impact of coping strategies on mental health while accounting for mental illness.

\subsection{Outlook and Directions for Future Research}

Categorizing coping strategies into generally helpful versus harmful seems unwarranted as the functionality of at least some strategies appears to be dynamic and influenced by contextual factors (e.g., Aldao and Tull 2015; Aldao and Wisco 2015; Catterson et al. 2017; De France and Evans 2020; Rottweiler et al. 2018). Yet, there are countless components of context, such as certain characteristics of the person executing the emotion regulation (e.g., sleep quality or perceived stress level), features of the emotion-eliciting stimuli/situations (e.g., anxiety due to moving to a new home versus anxiety due to chronic illness), implementation of regulatory processes, and types of outcomes (Aldao 2013) that have yet to be fully explored.

Identifying strategies that are always adaptive or always maladaptive also serves as a valuable contribution to psychotherapy research as conveying versus disputing certain coping strategies is a substantial part of many psychological treatment approaches. However, the situation may not be clear-cut as depressed mood may in fact be associated with engaging in many strategies - both supposedly adaptive and supposedly maladaptive-and not being able to successfully regulate one's response to stress, rather than with simply not engaging in adaptive coping.

While interventions aimed at fostering coping with everyday stressors have recently been developed for nonclinical populations such as students (e.g., Houston et al. 2017), more research is needed to determine whether stimulated use and non-use of coping strategies can directly translate into improved subjective well-being.

\subsection{Conclusion}

In conclusion, some coping strategies appear universally maladaptive or adaptive (e.g., catastrophizing), but for other coping strategies the impact on well-being depends on the situation (accepting versus attempting to change difficult situations). If replicated, this knowledge should be incorporated into psychotherapeutic treatment approaches that already target coping. Analyzing further if, when, and how coping strategies contribute positively to both mental health and well-being is essential for improving individuals' lives beyond reducing the detrimental effects of particular psychological disorders. 


\section{Appendix}

See Table 4.

Table 4 Psychiatric diagnoses screening

\begin{tabular}{lcc}
\hline Diagnosis & $\begin{array}{l}\text { Previously received } \\
n(\%)\end{array}$ & $\begin{array}{c}\text { WSQ } \\
n(\%)\end{array}$ \\
\hline $\begin{array}{l}\text { Attention Deficit Hyperactivity Disorder } \\
\text { Agoraphobia }\end{array}$ & $38(0.4)$ & $190(22.7)$ \\
Anxiety Disorder & $207(24.8)$ & $7(0.8)$ \\
Alcohol Use Disorder & $23(2.8)$ & \\
Bipolar Disorder & $261(31.2)$ & $67(8)$ \\
Depression & $29(3.5)$ & $44(5.3)$ \\
Eating Disorder & $32(3.8)$ & $114(13.6)$ \\
Generalized Anxiety Disorder & & $140(16.7)$ \\
Obsessive-Compulsive Disorder & $12(1.4)$ & $84(10)$ \\
Panic Disorder & & $405(48.4)$ \\
Panic Disorder+ Agoraphobia & & $324(38.8)$ \\
Personality Disorder & $67(8)$ & $488(58.4)$ \\
Specific Phobia & $12(1.4)$ & \\
Social Phobia & $33(3.9)$ & $50(6)$ \\
Post-Traumatic Stress Disorder & & \\
Schizophrenia/psychosis & & \\
Substance or alcohol use disorder & & \\
Suicidality & &
\end{tabular}

WSQ = Web Screening Questionnaire

Acknowledgement The authors would like to thank Marie Kühl and Swantje Borsutzky for their remarks on previous versions of this manuscript.

Funding Open Access funding enabled and organized by Projekt DEAL. This study received no external funding.

Availability of data and material Data will be made available upon reasonable request.

\section{Compliance with Ethical Standards}

Conflicts of interest The authors declare that they have no conflict of interest.

Consent to participate Informed consent was obtained from all individual participants included in the study.

Consent for publication All authors have approved the manuscript and its submission.

Ethics approval All procedures performed in studies involving human participants were in accordance with the ethical standards of the institutional and/or national research committee (the University Medical Center Hamburg-Eppendorf's ethics committee for psychological studies, LPEK-0026) and with the 1964 Helsinki declaration and its later amendments or comparable ethical standards. 
Open Access This article is licensed under a Creative Commons Attribution 4.0 International License, which permits use, sharing, adaptation, distribution and reproduction in any medium or format, as long as you give appropriate credit to the original author(s) and the source, provide a link to the Creative Commons licence, and indicate if changes were made. The images or other third party material in this article are included in the article's Creative Commons licence, unless indicated otherwise in a credit line to the material. If material is not included in the article's Creative Commons licence and your intended use is not permitted by statutory regulation or exceeds the permitted use, you will need to obtain permission directly from the copyright holder. To view a copy of this licence, visit http://creativecommons.org/licenses/by/4.0/.

\section{References}

Aldao, A. (2013). The future of emotion regulation research. Perspectives on Psychological Science, 8(2), 155-172. https://doi.org/10.1177/1745691612459518.

Aldao, A., \& Nolen-Hoeksema, S. (2012). When are adaptive strategies most predictive of psychopathology? Journal of Abnormal Psychology, 121(1), 276-281. https://doi.org/10.1037/a0023598.

Aldao, A., \& Tull, M. T. (2015). Putting emotion regulation in context. Current Opinion in Psychology, 3, 100-107. https://doi.org/10.1016/j.copsyc.2015.03.022.

Aldao, A., \& Wisco, B. E. (2015). Motivational conflict influences the timing of emotions and their regulation. Motivation and Emotion, 39(6), 943-952. https://doi.org/10.1007/s11031-015-9496-8.

Alvares, G. A., Quintana, D. S., Hickie, I. B., \& Guastella, A. J. (2016). Autonomic nervous system dysfunction in psychiatric disorders and the impact of psychotropic medications: A systematic review and meta-analysis. Journal of Psychiatry and Neuroscience, 41(2), 89-104. https://doi.org/10.1503/ jpn. 140217.

Arditte, K. A., Çek, D., Shaw, A. M., \& Timpano, K. R. (2016). The importance of assessing clinical phenomena in Mechanical Turk research. Psychological Assessment, 28(6), 684-691. https://doi. org/10.1037/pas0000217.

Balzarotti, S., Biassoni, F., Villani, D., Prunas, A., \& Velotti, P. (2016). Individual differences in cognitive emotion regulation: Implications for subjective and Psychological well-being. Journal of Happiness Studies, 17(1), 125-143. https://doi.org/10.1007/s10902-014-9587-3.

Baselmans, B. M., Willems, Y. E., van Beijsterveldt, C. E., Ligthart, L., Willemsen, G., Dolan, C. V., et al. (2018). Unraveling the genetic and environmental relationship between well-being and depressive symptoms throughout the lifespan. Frontiers in Psychiatry, 9, 1-12. https://doi.org/10.3389/fpsyt .2018.00261.

Broyd, A., Jolley, S., \& Johns, L. (2016). Determinants of subjective well-being in people with psychosis referred for psychological therapy in South London. British Journal of Clinical Psychology, 55(4), 429-440. https://doi.org/10.1111/bjc.12112.

Buhrmester, M. D., Talaifar, S., \& Gosling, S. D. (2018). An evaluation of Amazon's mechanical Turk, its rapid rise, and its effective use. Perspectives on Psychological Science, 13(2), 149-154. https://doi. org/10.1177/1745691617706516.

Catterson, A. D., Eldesouky, L., \& John, O. P. (2017). An experience sampling approach to emotion regulation: Situational suppression use and social hierarchy. Journal of Research in Personality, 69, 33-43. https://doi.org/10.1016/j.jrp.2016.04.004.

Chang, E. C., Yi, S., Liu, J., Kamble, S. V., Zhang, Y., Shi, B., et al. (2020). Coping behaviors as predictors of Hedonic well-being in Asian Indians: Does being optimistic still make a difference? Journal of Happiness Studies, 21(1), 289-304. https://doi.org/10.1007/s10902-019-00087-w.

De France, K., \& Evans, G. W. (2020). Expanding context in the role of emotion regulation in mental health: How socioeconomic status (SES) and developmental stage matter. Emotion. Advance online publication. https://doi.org/10.1037/emo0000743

Diener, E., Wirtz, D., Tov, W., Kim-Prieto, C., Choi, D., Oishi, S., \& Biswas-Diener, R. (2010). New wellbeing measures: Short scales to assess flourishing and positive and negative feelings. Social Indicators Research, 97(2), 143-156. https://doi.org/10.1007/s11205-009-9493-y.

Diener, E., Lucas, R. E., \& Oishi, S. (2018). Advances and open questions in the science of subjective wellbeing. Collabra: Psychology, 4(1), 15. https://doi.org/10.1525/collabra.115

Donker, T., van Straten, A., Marks, I., \& Cuijpers, P. (2009). A brief Web-based screening questionnaire for common mental disorders: development and validation. Journal of Medical Internet Research, 11(3), 19. https://doi.org/10.2196/jmir.1134. 
Dryman, M. T., \& Heimberg, R. G. (2018). Emotion regulation in social anxiety and depression: a systematic review of expressive suppression and cognitive reappraisal. Clinical Psychology Review, 65, 17-42. https://doi.org/10.1016/j.cpr.2018.07.004.

English, T., Lee, I. A., John, O. P., \& Gross, J. J. (2017). Emotion regulation strategy selection in daily life: The role of social context and goals. Motivation and Emotion, 41(2), 230-242. https://doi.org/10.1007/ s11031-016-9597-z.

Farrell, L. (2018). Understanding the relationship between subjective wellbeing and Gambling behavior. Journal of Gambling Studies, 34(1), 55-71. https://doi.org/10.1007/s10899-017-9692-4.

Franken, K., Lamers, S. M. A., Ten Klooster, P. M., Bohlmeijer, E. T., \& Westerhof, G. J. (2018). Validation of the Mental Health Continuum-Short Form and the dual continua model of well-being and psychopathology in an adult mental health setting. Journal of Clinical Psychology, 74(12), 2187-2202. https:// doi.org/10.1002/jclp.22659.

Gross, J. J. (2015). Emotion regulation: Current status and future prospects. Psychological Inquiry, 26(1), 1-26. https://doi.org/10.1080/1047840X.2014.940781.

Houston, J. B., First, J., Spialek, M. L., Sorenson, M. E., Mills-Sandoval, T., Lockett, M., et al. (2017). Randomized controlled trial of the Resilience and Coping Intervention (RCI) with undergraduate university students. Journal of American College Health, 65(1), 1-9. https://doi.org/10.1080/07448 481.2016.1227826.

Joormann, J., \& Stanton, C. H. (2016). Examining emotion regulation in depression: A review and future directions. Behaviour Research and Therapy, 86, 35-49. https://doi.org/10.1016/j.brat.2016.07.007.

Keyes, C. L. (2005). Mental illness and/or mental health? Investigating axioms of the complete state model of health. Journal of Consulting and Clinical Psychology, 73(3), 539. https://doi. org/10.1037/0022-006X.73.3.539.

Keyes, C. L. M. (2007). Promoting and protecting mental health as flourishing: A complementary strategy for improving National Mental Health. American Psychologist, 62(2), 95-108. https://doi. org/10.1037/0003-066X.62.2.95.

Kocalevent, R. D., Hinz, A., \& Brähler, E. (2013). Standardization of the depression screener Patient Health Questionnaire (PHQ-9) in the general population. General Hospital Psychiatry, 35(5), 551-555. https ://doi.org/10.1016/j.genhosppsych.2013.04.006.

Korpela, K. M., Pasanen, T., Repo, V., Hartig, T., Staats, H., Scopelliti, M., et al. (2018). Environmental strategies of affect regulation and their associations with subjective well-being. Frontiers in Psychology, 9, 1-15. https://doi.org/10.3389/fpsyg.2018.00562.

Kroenke, K., \& Spitzer, R. L. (2002). The PHQ-9: A new depression diagnostic and severity measure. Psychiatric Annals, 32(9), 509-515. https://doi.org/10.3928/0048-5713-20020901-06.

Kroenke, K., Spitzer, R. L., \& Williams, J. B. W. (2001). The PHQ-9. Journal of General Internal Medicine, 16(9), 606-613. https://doi.org/10.1046/j.1525-1497.2001.016009606.x.

Lazarus, R., \& Folkman, S. (1984). Stress, appraisal, and coping. Cham:Springer publishing company.

Liu, D. Y., \& Thompson, R. J. (2017). Selection and implementation of emotion regulation strategies in major depressive disorder: An integrative review. Clinical Psychology Review, 57, 183-194. https:// doi.org/10.1016/j.cpr.2017.07.004.

Manea, L., Gilbody, S., \& McMillan, D. (2012). Optimal cut-off score for diagnosing depression with the Patient Health Questionnaire (PHQ-9): a meta-analysis. CMAJ : Canadian Medical Association Journal = Journal de l'Association Medicale Canadienne, 184(3), 191-196. https://doi.org/10.1503/ cmaj.110829

Moritz, S., Jahns, A. K., Schröder, J., Berger, T., Lincoln, T. M., Klein, J. P., \& Göritz, A. S. (2016). More adaptive versus less maladaptive coping: What is more predictive of symptom severity? Development of a new scale to investigate coping profiles across different psychopathological syndromes. Journal of Affective Disorders, 191, 300-307. https://doi.org/10.1016/j.jad.2015.11.027.

Moritz, S., Fink, J., Miegel, F., Nitsche, K., Kraft, V., Tonn, P., \& Jelinek, L. (2018). Obsessive-compulsive disorder is characterized by a lack of adaptive coping rather than an excess of maladaptive coping. Cognitive Therapy and Research, 42(5), 650-660. https://doi.org/10.1007/s10608-018-9902-0.

Oppenheimer, D. M., Meyvis, T., \& Davidenko, N. (2009). Instructional manipulation checks: Detecting satisficing to increase statistical power. Journal of Experimental Social Psychology, 45(4), 867-872. https://doi.org/10.1016/j.jesp.2009.03.009.

Pavot, W., Diener, E., \& Suh, E. (1998). The temporal satisfaction with life scale. Journal of Personality Assessment, 70(2), 340-354. https://doi.org/10.1207/s15327752jpa7002_11.

Rottweiler, A.-L., Taxer, J. L., \& Nett, U. E. (2018). Context matters in the effectiveness of emotion regulation strategies. AERA Open, 4(2), 1-13. https://doi.org/10.1177/2332858418778849. 
Schiweck, C., Piette, D., Berckmans, D., Claes, S., \& Vrieze, E. (2019). Heart rate and high frequency heart rate variability during stress as biomarker for clinical depression. A systematic review. Psychological Medicine,, 49(2), 200-211. https://doi.org/10.1017/S0033291718001988.

Seligman, M. E. (2018). Positive psychology: A personal history. Annual Review of Clinical Psychology, 15(1), 1-23. https://doi.org/10.1146/annurev-clinpsy-050718-095653.

Tang, Y., \& Huang, Y. (2019). Contextual factors influence the selection of specific and broad types of emotion regulation strategies. British Journal of Social Psychology, 58(4), 1008-1033. https://doi. org/10.1111/bjso.12313.

van Ballegooijen, W., Riper, H., Cuijpers, P., van Oppen, P., \& Smit, J. H. (2016). Validation of online psychometric instruments for common mental health disorders: a systematic review. BMC Psychiatry, 16(1), 1-12. https://doi.org/10.1186/s12888-016-0735-7.

van Dijk, F., Schirmbeck, F., Boyette, L., \& de Haan, L. (2019). Coping styles mediate the association between negative life events and subjective well-being in patients with non-affective psychotic disorders and their siblings. Psychiatry Research, 272, 296-303. https://doi.org/10.1016/j.psych res.2018.12.020.

Westphal, M., Aldao, A., \& Jackson, C. (2017). Emotion dysregulation in comorbid posttraumatic stress disorder and substance use disorders: A narrative review. Military Psychology, 29(3), 216-233. https:// doi.org/10.1037/mil0000157.

U.S. Census Bureau (2020, March 30). Educational Attainment in the United States: 2019. Retrieved from https://www.census.gov/data/tables/2019/demo/educational-attainment/cps-detailed-tables.html

Publisher's Note Springer Nature remains neutral with regard to jurisdictional claims in published maps and institutional affiliations. 